\title{
A simple expression for vertical convective fluxes in planetary atmospheres
}

\author{
Ralph D. Lorenz ${ }^{\mathrm{a}, *}$ and Christopher P. McKay ${ }^{\mathrm{b}}$ \\ a Lunar and Planetary Laboratory, University of Arizona, Tucson, AZ 85721, USA \\ b NASA Ames Research Center Moffett Field, CA 94035, USA
}

Received 20 December 2002; revised 28 May 2003

\begin{abstract}
We explore the vertical convective flux $F_{C}$ in a radiative-convective grey atmosphere. An expression of the form $F_{C}=F_{S} \tau_{o} /\left(C+D \tau_{o}\right)$ appears useful, where $F_{S}$ is the shortwave flux absorbed at the base of an atmosphere with longwave optical depth $\tau_{O}$ and $C$ and $D$ are constants. We find excellent agreement with an idealized grey radiative-convective model with no shortwave absorption for $D=1$ and $C=1 \sim 2$ depending on the surface-atmosphere temperature contrast and on the imposed critical lapse rate. Where shortwave absorption is correlated with longwave opacity, as in the atmospheres of Earth and Titan, $C=2, D=2$ provides an excellent fit, validated against the present terrestrial situation and the results of a nongrey model of Titan's strongly antigreenhouse atmosphere under a wide range of conditions. The expression may be useful for studying the energetics of planetary climates through time where there is insufficient data to constrain more elaborate models.
\end{abstract}

(c) 2003 Elsevier Inc. All rights reserved.

Keywords: Atmospheres, dynamics; Radiative transfer; Prebiotic environments

\section{Introduction}

Vertical convection in planetary atmospheres is the driving force of weather. It is therefore perhaps surprising that it is rarely reported in model studies of planetary climate. Here we consider simple expressions that evaluate the heat flux transported by convection as a function of the radiative setting. These expressions will be useful in predicting the vigour of hydrological cycles on Titan, Mars, Paleo-Earths, and extrasolar planets. Vertical convection is also associated with the formation of clouds, and the maintenance of cloud opacity against gravity. Additionally, lightning (which may have an important role in prebiotic chemistry) is driven by vertical convection.

Although many terrestrial models now include convective parametrizations of varying sophistication (see, e.g., Emanuel, 1994) most models of the vertical structure of planetary atmospheres are radiative-convective models of the 'convective adjustment' type (Ramanathan and Coak-

\footnotetext{
* Corresponding author.

E-mail address: rlorenz@1pl.arizona.edu (R.D. Lorenz).
}

ley, 1978; see also, e.g., Chamberlain and Hunten, 1987; Goody and Yung, 1989). These models often do not explicitly report, nor does their associated literature usually present, the convective heat flux. This is considered an internal parameter that is simply used to adjust the model to remain below some specified critical lapse rate. This critical lapse rate is sometimes taken as the dry adiabat, although more typically for terrestrial models, the global average lapse rate of $6.5 \mathrm{~K} / \mathrm{km}$ (rather less than the dry adiabat of $10 \mathrm{~K} / \mathrm{km}$ ) is assumed. This choice represents a certain 'tuning' of the models that may in some cases obscure the underlying phenomena and resultant effects, many of which (e.g., viscous dissipation, see Gierasch, 1971) deserve further exploration.

In this paper, we explore the vertical convective heat flux required by a grey radiative-convective model, and compare firstly with an analytic expression derived from the Eddington approximation and secondly with a similar, but purely heuristic expression. We compare the latter with the results of a detailed nongrey antigreenhouse radiative-convective model for Titan, and with the known fluxes for Earth and Mars. 


\section{Grey radiative model}

We develop a simple grey radiative-convective model as follows. First, the conventional radiative profile of atmospheric temperature $T(\tau)$ and surface temperature $T_{s}\left(\tau_{o}\right)$ is generated, according to the Eddington approximation, thus

$T(\tau)=\sigma T_{e}^{4}(0.5+0.75 \tau)$

and

$\sigma T_{s}^{4}\left(\tau_{o}\right)=\sigma T_{e}^{4}\left(1.0+0.75 \tau_{o}\right)$,

where $\tau$ is the infrared grey optical depth and $\tau_{o}$ is the value at the surface, $\sigma$ the Stefan-Boltzmann Constant, $T_{e}$ the effective temperature of the planet, with $\sigma T_{e}^{4}=F_{s}$ where $F_{s}$ is the diurnally-averaged solar flux deposited into the surface. This can be related to the top-of-atmosphere solar constant $F_{o}$ by an expression of the form $F_{s}=0.25 F_{o}(1-A) \times$ $\exp \left(-\tau_{s} / \cos \mu_{o}\right)$ for an atmosphere with shortwave absorbers of optical depth $\tau_{s}$, or $F_{s}=0.25 F_{o}(1-A)[1 /(1+$ $\left.\tau_{s} / \cos \mu_{o}\right)$ ] for shortwave scatterers, with $A$ being the planetary albedo. $\mu_{o}$ is the mean solar zenith angle, or $\arccos (0.5)$.

The radiative equilibrium introduces a strong temperature discontinuity at the surface. Although discussions of convection often focus on the $T(\tau)$ profile exceeding an imposed critical lapse rate, we note here that in purely radiative equilibrium, this discontinuity is responsible for the rejection of much of the heat deposited at the surface (especially in optically-thin atmospheres), and the elimination of the discontinuity in a radiative-convective model therefore requires that heat flux to be transported by convection.

The optical depth may be specified as a function of altitude $z$ as $\tau(z)=\tau_{o} \exp \left(-z / H_{\tau}\right)$ where $H_{\tau}$ is a scale height that relates to the source of the opacity (note that in general this opacity scale height may not be the same as the pressure scale height $H$ ). We can similarly specify the pressure profile as $P(z)=P_{0} \exp (-z / H)$ with $P_{0}$ the surface pressure and $H$ is the pressure scale height.

In some situations (e.g., McKay et al., 1999) it is instructive or convenient to specify the optical depth as a function of pressure, e.g., $\tau=\tau_{0}\left(P / P_{0}\right)^{n}$, where $n=1$ might represent a simple absorber that is uniformly mixed in the atmosphere, or $n=2$ a source of collision-induced opacity. To reconcile with our formulations above, the cases of $n=1$ and 2 can be expressed simply by setting $H_{\tau}=H$ or $H_{\tau}=H / 2$.

In the terrestrial atmosphere, where water vapor is the dominant absorber, the scale height of the optical depth is much shorter (typically assumed to be $2-3 \mathrm{~km}$ ) than the background atmosphere $(\sim 8 \mathrm{~km})$, given for an ideal isothermal gas as $H=R_{O} T / g M$ where $R_{O}$ is the universal gas constant $8314 \mathrm{~J} \mathrm{~kg}^{-1} \mathrm{~K}^{-1}, T$ the absolute temperature, $g$ the acceleration due to gravity, and $M$ the relative molecular weight. Looked at another way, the terrestrial situation can be represented as $\tau=\tau_{0}\left(P / P_{0}\right)^{n}$ with $n \sim 4$.

\section{Convective adjustment}

We perform the conventional convective adjustment to the model by specifying a critical lapse rate $\Gamma$. We leave this as an arbitrary parameter, noting that the typical value used for Earth is an empirical one, rather than the dry adiabatic value of $\Gamma_{a d}=g / C_{p}$, where $C_{p}$ is the specific heat capacity at constant pressure. We note again that convective adjustment may be required not only for the generally-noted condition that the radiative profile $T(z)$ exceeds $\Gamma$, but the surface discontinuity itself implies a locally infinite lapse rate and thus convective adjustment is, in some sense, always required.

Convective adjustment-fixing the lapse rate to be equal to $\Gamma$-is performed from some trial optical depth $\tau_{t}$ down to the surface, with the surface temperature held equal to-or offset by some $\Delta T$ above-that of the atmosphere immediately above the surface. $\tau_{t}$ must be adjusted such that the net IR flux upwelling from the atmosphere and surface below equals that of the radiative equilibrium profile. This upwelling flux will include two components-radiation from the surface, and radiation from the atmosphere. Imposing the continuity condition at the surface, and the specified lapse rate through the troposphere, in general decreases the surface and lower atmospheric contributions to this upwelling flux, while increasing the temperatures and thus the upwelling fluxes at higher levels in the troposphere. The transfer of flux from the surface (where it is deposited) to these higher levels is accomplished by convection. We calculate the convective flux as a function of altitude as the imbalance of radiative fluxes. Specifically, at a given optical depth $\tau, F_{u p \_s u r f}$ is upwelling IR flux emitted from the surface and attenuated by the atmosphere $\left(\tau_{o}-\tau\right), F_{\text {down }}$ is IR flux radiated down from the atmosphere above. $F_{u p}$ is the upwelling IR flux from the atmosphere- this starts from zero near the surface (with a vanishingly thin radiating layer) to a maximum in the first optical depth or so as seen from the surface where the atmosphere is warmest, down to equal $F_{S}$ at the top of the atmosphere.

$$
\begin{aligned}
& F_{\text {up_surf }}(\tau)=\sigma T_{s}^{4} \exp \left[-\left(\tau_{o}-\tau\right) / \mu\right], \\
& F_{\text {down }}(\tau)=\int_{0}^{\tau} \sigma T^{4}(\tau) \exp \left[\left(\tau_{*}-\tau\right) / \mu\right] d \tau_{*}, \\
& F_{\text {up }}(\tau)=\int_{\tau_{0}}^{\tau} \sigma T^{4}(\tau) \exp \left[-\left(\tau_{*}-\tau\right) / \mu\right] \delta \tau_{*},
\end{aligned}
$$

$\tau_{*}$ in these expressions is a dummy variable of integration, and $\mu$ is the average cosine of the emission angle (0.66). The imbalance of these fluxes $F_{c}=F_{s}-F_{n e t}$, where $F_{n e t}=$ $F_{\text {up_surf }}+F_{\text {up }}-F_{\text {down }}$ is assumed to be transported by convection. These fluxes are computed for a terrestrial-like case in Fig. 1. Note that $F_{\text {down }}$ at the surface exceeds the incident solar flux $F_{S}$ - the warm atmosphere contributes an enormously important part of the surface energy balance. Little 


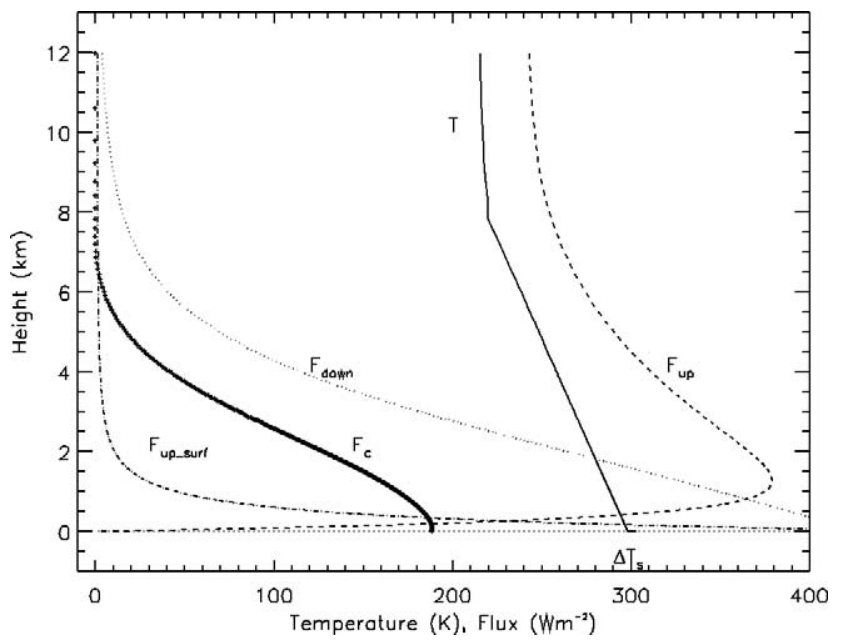

Fig. 1. Structure of a $1 \mathrm{D}$ radiative convective model with $F_{S}=240 \mathrm{~W} \mathrm{~m}^{-2}$, optical depth $\tau=4$, imposed lapse rate of $10 \mathrm{~K} / \mathrm{km}$, and surface temperature contrast $\Delta T=5 \mathrm{~K}$. The solid line shows the temperature profile; Dash-dot line $F_{u p \_s u r f}$ is upwelling IR flux emitted from the surface, dotted curve $F_{\text {down }}$ is IR flux radiated down from the atmosphere. The dashed curve $F_{u p}$ is the upwelling IR flux from the atmosphere-this starts from zero near the surface (with a vanishingly thin radiating layer) to a maximum in the first optical depth or so, down to equal $F_{S}$ at the top of the atmosphere. The Thick line of crosses is the net flux, assumed to be transported by convection, $F_{c}=F_{S}+F_{\text {down }}-F_{S_{-} u p}-F_{\text {up }}$.

of the radiation emitted from the surface $F_{u p \_s u r f}$ reaches the top of the atmosphere in a grey model (although in the nongrey real world, spectral windows allow surface emission to space). In general the convective flux increases monotonically with increasing depth, such that the maximum flux occurs at the surface. However, if a large surface temperature contrast is permitted, the large $F_{u p \_s u r f}$ permitted in the lowest optical depth of the atmosphere can reject much of the heat from the surface and near-surface, such that the vertical convective flux actually peaks at some altitude above the surface. However, the temperature contrasts required to do that may not always be physical, since the large local temperature gradient would tend to promote stronger convective heat removal.

We show in Fig. 2 the resultant maximum convective flux as a function of $\tau_{o}$ for a terrestrial situation with $F_{S}=140$ $\mathrm{W} \mathrm{m}^{-2}$ and $H \sim 10 \mathrm{~km}, H_{t} \sim 2 \mathrm{~km}$. We impose dry adiabatic lapse rate of $9.5 \mathrm{~K} / \mathrm{km}$ and the more typical value (influenced largely by moist effects) of $6.5 \mathrm{~K} / \mathrm{km}$, and surface temperature contrasts of 0 and $5 \mathrm{~K}$. We note that Lindzen et al. (1982) find $\Delta T=0 \sim 6 \mathrm{~K}$ in a radiative-convective model with a cumulus parameterization of convection, and $\Delta T \sim 2 \mathrm{~K}$ for adjustment models; Pujol and Fort (2002) explore the surface temperature contrast and its influence on entropy production by convection. As one might expect, the higher the optical depth, the higher the convective flux. Similarly, the steeper the imposed lapse rate, or the larger the surface temperature contrast, the better the system can reject heat by radiation, and thus the lower the convective heat flux.

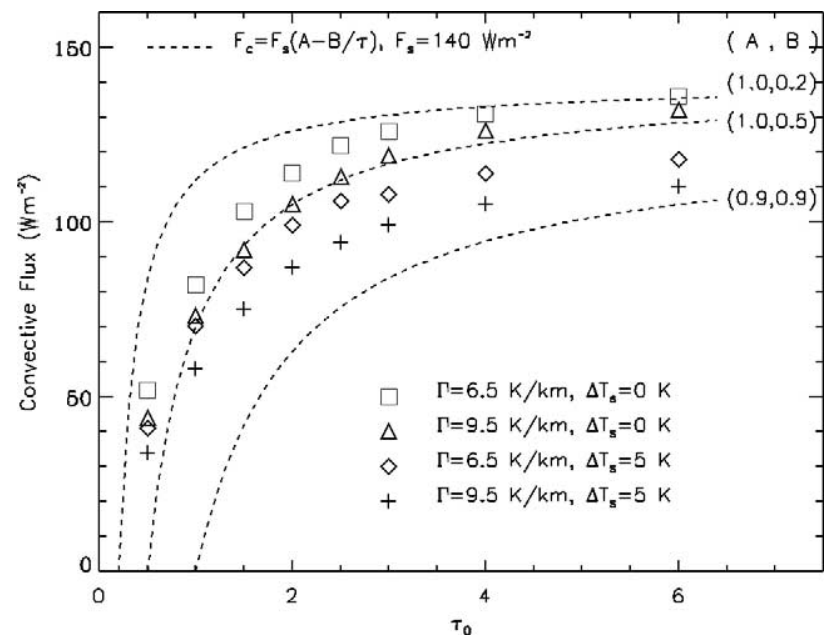

(a)

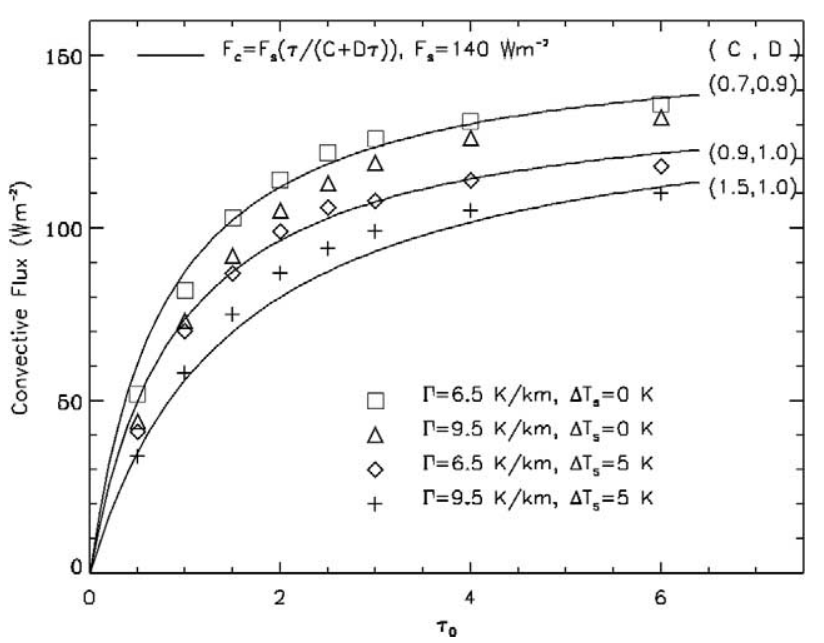

(b)

Fig. 2. Maximum convective flux (symbols) in grey radiative-convective models with varying infrared optical depth $\tau$, with various specified critical lapse rates and surface temperature contrasts. The two panels show two sets of representative analytic expressions: (a) of the form $A-B / \tau$, following equation in the text, and (b) a heuristic form $\tau /(C+D \tau)$ which passes through the origin.

\section{Analytic expressions}

For a dry adiabatic atmosphere, we can write the lapse rate in another way (see also Yokohata et al., 2002), specifically as $d\{\log T\} / d\{\log P\}=L$ with $L=R_{0} / C_{p} M$ where $C_{p}$ is the specific heat at constant pressure $\left(\sim 1000 \mathrm{~J} \mathrm{~kg} \mathrm{~K}^{-1}\right.$ for nitrogen) and thus $L \sim 0.287$. Since we can also write $\tau$ as a function of $P$ above, we can write in the troposphere $T=T_{t}\left(\tau / \tau_{t}\right)^{L / n}$. Furthermore, since the net radiative flux due to the atmosphere is given by $(4 \sigma / 3)\left(d T^{4} / d \tau\right)$, we can thus write for the convective flux due to the atmosphere $F_{c a}$

$F_{c a}=F_{s}\left[1-\left(\frac{R}{C_{p}}\right) \frac{1+1.5 \tau}{0.375 n \tau}\right]$,

where $R$ is the specific gas constant $R_{o} / M$. If we impose a condition of no temperature contrast at the surface, we must 
add the to the atmospheric convective flux the radiative flux that would otherwise come from the temperature discontinuity at the surface, namely $\left(\sigma T_{s}^{4}-\sigma T_{\tau o}^{4}\right)=0.5 \sigma T_{s}^{4}=0.5 F_{s}$, to yield the total convective flux thus

$F_{c}=F_{s}\left[1.5-\left(\frac{R}{C_{p}}\right) \frac{1+1.5 \tau}{0.375 n \tau}\right]$.

This can in turn be written in the form

$F_{c}=F_{s}(A-B / \tau)$,

where $A=1.5-(4 / n)\left(R / C_{p}\right)$ and for nitrogen we get $A=0.32,0.9,1.2$ for values of $n=1,2,4$. Note that without the extra 0.5 term in $A$ due to the surface discontinuity, the expression would be always negative (implying unphysical downward convection) for $n=1$ or less-see also Sagan (1969)—convection in a free atmosphere requires nonlinear variation of opacity with pressure. The parameter $B=(8 / 3 n)\left(R / C_{p}\right)=0.765,0.4,0.2$ for $n=1,2,4$ indicates the extent to which the convective flux increases with increasing optical depth-the thicker the atmosphere, the harder it is for flux to leave it radiatively and thus the more convective flux there must be. Note that substantially different values result for water vapor, with $\left(R / C_{p}\right) \sim 0.46$ : $A=-0.344,0.578,1.039, B=1.23,0.61,0.41$.

Figure 2a plots Eq. (8) above against the radiativeconvective results. Although equations of this form can give somewhat representative fits, the parameters that do so do not represent the choices one would expect from the purely theoretical considerations above. For example, since $n \sim 4$, we would expect $A \sim 1, B=0.2-0.4$, which yields a rather weak dependence on $\tau$. Furthermore, the tendency of all the expressions to become negative at low $\tau$ is unphysical.

Heuristically, we can force the convective flux to be zero at $\tau_{o}=0$, and preserve the asymptotic character of the flux tending to a constant value as $\tau_{o}$ tends to infinity with an expression of the form

$F_{c}=F_{s}\left[\tau_{o} /\left(C+D \tau_{o}\right)\right]$.

Equations of this form are plotted in Fig. 2b, and show an excellent fit with $D \sim 1$ and $C$ depending on the lapse rate and discontinuity. Although we propose this equation on purely heuristic grounds, we will note that the lapse rate $d T / d z$ in a radiative atmosphere is (Ramanathan and Coakley, 1978)

$d T / d z=(g / 4 R) F_{r} \tau /\left(F_{r} \tau+c\right)$,

where $g$ is gravitational acceleration, $R$ the gas constant, $F_{r}$ the net upward radiative flux and $c$ a constant. For the lapse rate to be positive, it follows that $d\left(F_{r} \tau\right) / d \tau>0$, or if we arbitrarily relate $F_{r}$ to $\tau$ as $F_{r} \sim \tau^{n}$, with $n<1$, or as $F_{r} \sim(\tau+1)^{n}$ with $n \leqslant 1$. If we take this latter expression, with $n=1$, then since $F_{c}=F_{s}-F_{r}$, it follows that $0 \leqslant F_{c} \leqslant F_{s} \tau /(1+\tau)$. Our Eq. (9) above satisfies this inequality, provided $(C+D \tau)>(1+\tau)$, which is always the case if $C$ and $D$ are greater than or equal to 1 .

The typical convective flux for the Earth is $80-100 \mathrm{~W} \mathrm{~m}^{-2}$ compared with the absorbed solar flux of $240 \mathrm{~W} \mathrm{~m}^{-2}$.
While Eq. (9) with $C=1 \sim 2, D=1$ agrees well with the shortwave-transparent model shown in Fig. 2, the real terrestrial situation has rather lower convective flux than the $\sim 160 \mathrm{~W} \mathrm{~m}^{-2}$ we would therefore predict. This discrepancy is due in part to the nongreyness of the real atmosphere (any window IR emission to space cannot participate in convection), and in part due to the fact that of the incident shortwave $240 \mathrm{~W} \mathrm{~m}^{-2}$ that is not reflected to space, some significant fraction is absorbed in the atmosphere. Previous model studies (e.g., Ozawa and Ohmura, 1997; Pujol and Fort, 2002) assume the shortwave opacity is related linearly with the infrared optical depth, and that only $\sim 140 \mathrm{~W} \mathrm{~m}^{-2}$ is absorbed at the surface. As well as reducing the flux available at the surface, this absorption warms the atmosphere and thus lowers the temperature gradient driving convection. Setting $F_{S}=140 \mathrm{~W} \mathrm{~m}^{-2}$ (following those studies) with $C=1 \sim 2$ and $D=1$ yields $F_{c}=93 \sim 112 \mathrm{~W} \mathrm{~m}^{-2}$, a satisfactory result. Pavlov (personal communication) suggests $F_{s}=170 \mathrm{~W} \mathrm{~m}^{-2}$ is an appropriate value from two terrestrial models which calculate $F_{c}=125 \mathrm{~W} \mathrm{~m}^{-2}$ : with this value of $F_{s}$, our model yields $113-136 \mathrm{~W} \mathrm{~m}^{-2}$ for $C=1 \sim 2$ and $D=1$, again a good agreement. Note that without more detailed information on $\tau_{s}$, for the present terrestrial atmosphere at least, the effects of shortwave absorption and scattering can be crudely simulated by setting $D=2$ and using the top-of-atmosphere flux, $F_{s}=240 \mathrm{~W} \mathrm{~m}^{-2} . D=2$ has the effect of suppressing the convective flux in the sense of correlating shortwave attenuation of $F_{s}$ with the infrared optical depth and yields, at least in this instance, results comparable to those above.

\section{Titan model}

A radiative-convective model of Titan's atmosphere (McKay et al., 1989, 1991) was used to further explore this problem. Variants of this model have also been used to retrieve haze and surface parameters from telescopic observations of Titan. It (McKay et al., 1993), and semiempirical fits to it (Lorenz et al., 1999), have also been used, coupled to a surface thermodynamic equilibrium model, to study variations in Titan's atmosphere through geologic time as photolysis and solar evolution have changed the volatile budget and the radiative environment.

The model is a conventional 1-dimensional radiative transfer code with convective adjustment which resolves the short and longwave fluxes into 21 and 33 wavelength regions, respectively. Haze microphysics is incorporated into the model. The convective flux at the ground is computed from the difference between the upwelling and downwelling radiative fluxes. For the present-day Titan, this corresponds to around $1 \%$ of the incident solar flux (only around $10 \%$ of the incident solar flux reaches the surface at all, in fact, owing to absorption of blue light by the haze, and to a lesser extent the absorption of some red and near-IR wavelengths by tropospheric methane). 
The model was run with a selection of input parameters, with a fixed total pressure and solar constant. First, surface methane humidity (a prime determinant of the greenhouse effect, although via red and near-IR absorption, it also controls the shortwave flux reaching the surface) was varied from 0 to $80 \%$ (with the present value believed to be $40-$ $60 \%$ ). Second, the hydrogen mixing ratio (hydrogen is a noncondensible greenhouse gas) was varied from 0 to 0.008 (a present value of 0.001 to 0.002). A geothermal flux of 0 to $0.4 \mathrm{~W} \mathrm{~m}^{-2}$ was introduced at the surface: the latter value is only appropriate for very early in Titan's history, but is useful in exploring the convection flux parameter space. Finally, the haze production was varied from the present value down to near zero-this controls the haze optical depth and thus the shortwave flux reaching the surface. Parameters which led to surface temperatures in excess of $110 \mathrm{~K}$, and thus with surface IR fluxes approaching double those at $94 \mathrm{~K}$, were not considered.

The Rosseland mean infrared opacity $\tau$ was computed from the model by integrating opacities over the infrared spectrum, and the flux deposited at the surface $F_{S}$ (equal to the shortwave flux absorbed at the surface, plus any geothermal heat flow) was recorded. The upward convective flux $F_{c}$ is calculated from the difference between the net infrared and shortwave + geothermal fluxes in the lowest level in the model.

We find (see Fig. 3) that Eq. (9) with $C=2, D=2$ (as for the Earth) shows reasonable agreement with the fluxes determined from the model for opacities of 0.3 to 6.5 , and surface temperatures of 80 to $110 \mathrm{~K}$. A subset of the data shows a slight offset from this line, but a very consistent slope.

Perfect agreement would not be expected in any case since the convective flux is estimated from the difference between the (much larger) radiative fluxes at the surface,

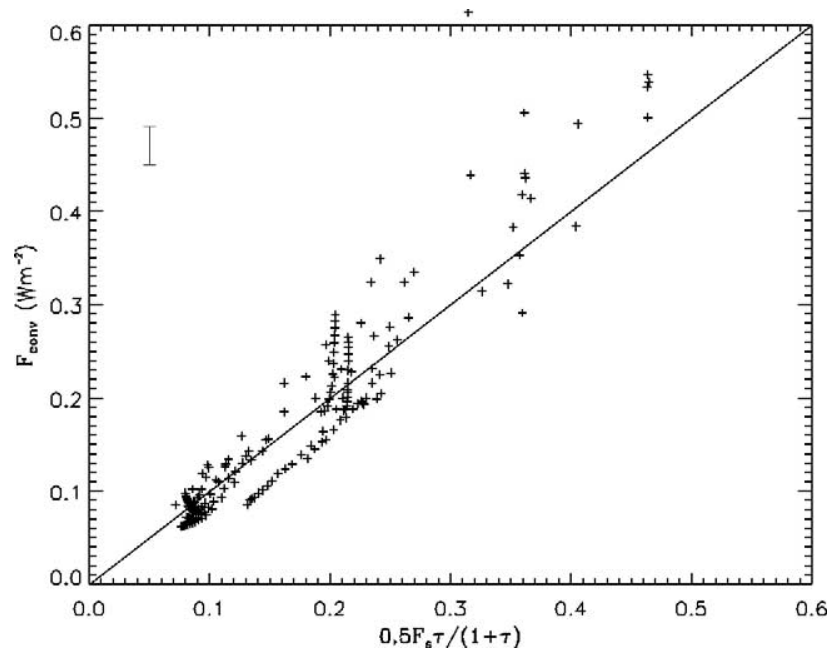

Fig. 3. Convective flux extracted from a nongrey Titan radiative-convective model with antigreenhouse effect, compared with simple estimate (Eq. (9) with $C, D=2$ ) based on shortwave heat flux to surface and a Rosseland mean optical depth. such that the estimated convective flux is unlikely to be accurate to better than some tens of percent in relative terms, or around $0.02 \mathrm{~W} \mathrm{~m}^{-2}$ in absolute terms $(0.5 \%$ of the incident solar flux). Furthermore, there is a window at around $530 \mathrm{~cm}^{-1}$ which permits nongrey emission from the surface.

\section{Discussion}

Although we offer Eq. (9) with no further justification than that it adequately describes the available results, it is of note that this formulation shows how increased opacity forces more heat flux through convection. In an electrical analogy with current corresponding to heat flow, convection and radiation are two parallel current paths, with the radiative 'resistance' corresponding to optical depth. The voltage driving the current through the two resistors corresponds to the difference between surface temperature and effective temperature (at which heat is rejected from the system). In the case of $\tau=0$, there is no radiative resistance and no temperature difference, so no power can be extracted and hence no convective heat flux. For high values of $\tau$, there is substantial resistance to the flow of heat by radiation and the surface temperature increases. Decreasing the convective resistance up to a point increases the current (heat flux) transmitted by convection, but reduces the surface temperature.

A slightly more elaborate and representative model is sketched in Fig. 4, with the radiative resistance broken down into two parts, only one of which is shorted by a convective path. Current $i$ corresponds to the deposited solar flux $\left(F_{S}\right)$. The stratospheric and tropospheric resistances $R_{S}$ and $R_{t}$ correspond to the infrared opacities there (relating to approximately 1 and $\tau_{o}-1$, respectively). Resistance $R_{c}$ corresponds to convection 'shorting out' the tropospheric radiative resistance $R_{t}$. Voltages $V_{s}$ and $V_{t}$ correspond to surface and tropopause temperatures, respectively. By Ohm's law, $V_{t}=i R_{s}$ and $V_{s}=i\left(R_{s}+1 /\left(1 / R_{t}+1 / R_{c}\right)\right)$. The convective heat flux is represented by the current through $R_{c}$, or $i\left(R_{t} /\left(R_{t}+R_{c}\right)\right)$, echoing the form of our expression $F_{s}(\tau / C+D \tau)$.

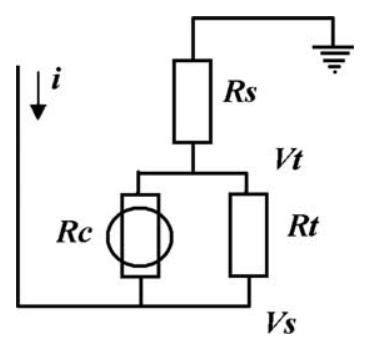

Fig. 4. Electrical circuit analog of radiative-convective equilibrium. Shortwave flux reaching the ground corresponds to current $i$. Stratospheric and Tropospheric resistances correspond to opacities (approximately 1 and $\tau_{o}-1$ respectively for optically-thick atmospheres). Resistance $R_{C}$ corresponds to convection 'shorting out' the radiative resistance $R_{t}$. Voltages $V_{S}$ and $V_{t}$ correspond to surface and tropopause temperatures, respectively. 
The question now becomes one of how to select $R_{c}$. In a well-understood system, this might be derived from dynamical simulations. In the conventional approach of convective adjustment, $R_{c}$ is replaced by a diode, with a fixed voltage drop-supply of additional current or increasing $R_{t}$ simply forces more current through that element, such that the convective heat flux always maintains a constant lapse rate or temperature difference.

Another approach may be to select the convective 'resistance' to maximise the electrical power dissipated in that element-i.e., the product of voltage difference and current flow. There is some evidence (Lorenz, 2003; Paltridge, 1975) that horizontal heat transport on the Earth may adjust itself to maximize dissipation (or entropy production), apparently also the case with Titan and Mars (Lorenz et al., 2001). Ozawa and Ohmura (1997) present a $1 \mathrm{D}$ radiative-convection model (with shortwave absorption) with the vertical convective flux selected to maximize entropy production-without regard to any critical lapse rate-and find that the observed terrestrial atmosphere seems consistent with this idea. It is of interest that the predicted convective fluxes $[54,77,89,96,101] \mathrm{W} \mathrm{m}^{-2}$ for $\tau=[1,2,3,4,5]$ are exactly in agreement with our Eq. (9) with $C=2, D=2$, and $F_{s}=240 \mathrm{~W} \mathrm{~m}^{-2}$.

As a final crude test for our expression, we note that the present-day greenhouse effect on Mars is about $2 \mathrm{~K}$ with $\tau \sim 0.1$ and $F_{S} \sim 120 \mathrm{~W} \mathrm{~m}^{-2}$, and hence from Eq. (9) we would predict a convective heat flow of only $\sim 6 \mathrm{~W} \mathrm{~m}^{-2}$. This appears to be quite consistent with measurements made by Mars landers (e.g., Sutton et al., 1978), suggesting an afternoon peak flux of $\sim 20 \mathrm{~W} \mathrm{~m}^{-2}$, or only about $5 \mathrm{~W} \mathrm{~m}^{-2}$ averaged over the entire day.

Detailed information on the convective fluxes in giant planet tropospheres is not available, but one may assume that their deep interiors are characterized by very large optical depths. Setting $\tau$ as infinity and substituting an internal heat flow for $F_{S}$ in our expression suggests all the internal heat flow is removed by convection at depth-only when small optical depths are reached does the emerging heat flux begin to become radiative. This is of course observed in the temperature profiles of the giant planets which become adiabatic at depth, consistent with heat transport dominated by convection.

We have essentially assumed that shortwave absorption plays no role in the troposphere, other than to reduce the flux that reaches the surface. In the case of atmospheres like Titan, where the shortwave opacity is predominantly at altitudes above the infrared opacity, this is straightforward. The atmosphere of the early Earth may have had a similar high-altitude haze (e.g., Pavlov et al., 2001) formed by the photolysis of methane. The fact that high-altitude absorption warms the stratosphere (thereby in principle warming the tropopause and thus reducing the temperature difference that drives convection) does not appear to make the convective flux differ substantially from our estimate, at least in the Titan case. Note that analytic solutions for purely radia- tive situations where the short- and long-wave opacities have been treated in McKay et al. (1999).

A situation with shortwave absorption in the atmosphere that is mixed with the longwave opacity is explored in Ozawa and Ohmura (1997). The magnitudes of the convective fluxes are more or less the same as those reported here (taking the same value of $F_{S}$ ), but the shape of the convective flux profile is rather different - the flux is approximately constant from the surface up to where the infrared opacity is around unity, rather than declining sharply from the surface. In other words, the absorbed shortwave flux adds to the convective flux where it is absorbed (if the temperature profile is fixed to the critical lapse rate, the longwave fluxes must remain constant) and our estimate of the surface convective flux remains usefully accurate.

It may be, however, that there may be situations where shortwave absorption is mixed with the longwave absorption in such a way that our expression does not perform well for the surface (indeed, in these cases there may be substantial convection above the shortwave absorption but not below it -in such cases it may be prudent to use our expression as if the shortwave absorber acted as the surface). We note, however, that our approach appears successful for all known planetary atmospheres.

The further caveat we must point out is that our simple expression, like the radiative-convective equilibrium models it attempts to fit, is a steady-state model. There may be time-variant situations (either by virtue of seasonal or diurnal forcing of the shortwave flux, or perhaps by a periodic convection response) that are not described well by it. Such complex situations, by definition, are beyond the scope of this work.

\section{Conclusions}

We have investigated vertical convective fluxes in radiative-convective models, and find that an empirical equation of the form $F_{c}=F_{s} \tau_{o} /\left(C+D \tau_{o}\right)$ with $C, D=2$ yields a good estimate of the convective fluxes in all the real cases we have discussed, although $C=1 \sim 2, D=1$ is appropriate for idealized true grey cases with no shortwave absorption (or where such absorption is already taken into account in specifying $F_{S}$ ). In all cases, these expressions are good to a factor of $\sim 2$ or generally better, suggesting they have utility for investigating the fluxes and thus the vigor of hydrological cycles in theoretical investigations of planetary climate.

\section{Acknowledgments}

RDL acknowledges the support of the Cassini project and CPM that of the NASA Planetary Atmospheres Program. We thank Tokuto Yokohata and reviewer Alex Pavlov for useful discussion and comments. 


\section{References}

Chamberlain, J.W., Hunten, D.M., 1987. Theory of Planetary Atmospheres: An Introduction to their Physics and Chemistry. Academic Press, New York. 481 pp.

Emanuel, K.A., 1994. Atmospheric Convection. Oxford Univ. Press, Oxford.

Gierasch, P.J., 1971. Dissipation in atmospheres: the thermal structure of the martian lower atmosphere with and without viscous dissipation. J. Atmos. Sci. 28, 315-324.

Goody, R.M., Yung, Y.L., 1989. Atmospheric Radiation: Theoretical Basis, 2nd edition. Oxford Univ. Press, Oxford. 519 pp.

Lindzen, R.S., Hou, A.Y., Farrell, B.F., 1982. The role of convective model choice in calculating the climate impact of doubling $\mathrm{CO}_{2}$. J. Atmos. Sci. 39, 1189-1205.

Lorenz, R.D., 2003. Full steam ahead-probably. Science 299, 837-838

Lorenz, R.D., McKay, C.P., Lunine, J.I., 1999. Analytic stability of Titan's climate: sensitivity to volatile inventory. Planet. Space Sci. 47, 15031515.

Lorenz, R.D., Lunine, J.I., Withers, P.G., McKay, C.P., 2001. Entropy production by latitudinal heat transport on Titan, Mars and Earth. Geophys. Res. Lett. 28, 415-418.

McKay, C.P., Pollack, J.B., Courtin, R., 1989. The thermal structure of Titan's atmosphere. Icarus 80, 23-53.

McKay, C.P., Pollack, J.B., Courtin, R., 1991. The greenhouse and antigreenhouse effect on Titan. Science 253, 1118-1121.
McKay, C.P., Pollack, J.B., Lunine, J.I., Courtin, R., 1993. Coupled atmosphere-ocean models of Titan's past. Icarus 102, 88-98.

McKay, C.P., Lorenz, R.D., Lunine, J.I., 1999. Analytic models of the greenhouse and anti-greenhouse effects: application to Titan and the early Earth. Icarus 137, 57-61.

Ozawa, H., Ohmura, A., 1997. Thermodynamics of a global-mean state of the atmosphere: a state of maximum entropy increase. J. Climate 10, 441-445.

Paltridge, G.W., 1975. Global dynamics and climate change: a system of minimum entropy exchange. Q. J. Roy. Meteor. Soc. 101, 475-484.

Pavlov, A.A., Brown, L.L., Kasting, J.F., 2001. UV shielding of $\mathrm{NH}_{3}$ and $\mathrm{O}_{2}$ by organic hazes in the archean atmosphere. J. Geophys. Res. 106, 23267-23288.

Pujol, T., Fort, J., 2002. States of maximum entropy production in a onedimensional vertical model with convective adjustment. Tellus Ser. A 54, 363-369.

Ramanathan, V., Coakley Jr., J.A., 1978. Climate modeling through radiative-convective models. Rev. Geophys. Space Phys. 16, 465-489.

Sagan, C., 1969. Gray and nongray planetary atmospheres: structure, convective instability and greenhouse effect. Icarus 10, 290-300.

Sutton, J.L., Leovy, C.B., Tilman, J.E., 1978. Diurnal variations of the martian surface layer meteorological parameters during the first 45 sols at two Viking lander sites. J. Atmos. Sci. 34, 2346-2355.

Yokohata, T., Kuramoto, K., Odaka, M., 2002. Role of $\mathrm{H}_{2} \mathrm{O}$ and $\mathrm{CO}_{2}$ ices in martian climate changes. Icarus 159, 439-448. 